\title{
Alteración de la visión y su relación con la utilización de aparatos electrónicos en adolescentes de escuelas públicas de San Lorenzo
}

\author{
Alteration of vision and its relationship with the use of \\ electronic devices in adolescents attending public schools in \\ San Lorenzo
}

\author{
Jessica Ferreira Martínez', Olivia Cardozo'
}

\section{RESUMEN}

Introducción: Los defectos de refracción no corregidos son la principal causa de discapacidad visual. En sitios en donde la atención oftalmológica es escasa, se han realizado programas de tamizaje por los maestros, que fueron eficientes para detección precoz de defectos refractivos. Objetivo: Determinar la frecuencia de alteración de la visión y su relación con la utilización de aparatos electrónicos en adolescentes de dos escuelas de San Lorenzo. Materiales y Métodos: Estudio observacional, descriptivo, con componente analítico. Fueron incluidos adolescentes de 12 a 18 años de edad de dos escuelas de San Lorenzo. Las variables estudiadas fueron: Edad, Sexo, visión menor a 20/40, antecedente de consulta previa, antecedente familiar de uso de anteojos, horas de uso de aparatos electrónicos, uso de redes sociales, horas al aire libre, tiempo dedicado a lectura no académica, presencia de síntomas oculares. Análisis estadístico: SPSSv21 utilizando estadística descriptiva e inferencial. Protocolo aprobado por el comité de ética institucional con consentimiento informado. Resultados: fueron evaluados 300 adolescentes, se detectó que la mediana de edad fue 14 años, predominó el sexo femenino (58,7\%); el 7,3\% presentó una alteración de la visión, el $64,3 \%$ no consultó previamente. El antecedente familiar de uso de anteojos, no tuvo significancia. Se vio que las horas diarias de uso de aparatos electrónicos fue mayor en los que tenían visión disminuida con un menor tiempo al aire libre en aquellos con visión alterada. El diagnóstico más frecuente fue astigmatismo. Conclusión: La frecuencia de alteración de la visión en adolescentes fue 7,3\%. Los adolescentes con visión alterada tuvieron más horas diarias al uso de aparatos electrónicos, observándose que las horas que pasaban al aire libre fue menor en los mismos.

Palabras claves: Visión, aparatos electrónicos, vicios de refracción.

\section{ABSTRACT}

Introduction: Uncorrected refractive errors are the main cause of visual impairment. In places where ophthalmological care is poor, screening programs have been carried out by teachers, which were efficient for early detection of refractive errors. Objective: To determine the frequency of vision impairment and its relationship with the use of electronic devices in adolescents of two schools in San Lorenzo. Materials and Methods: This was an observational and descriptive study, with an analytical component. Teenagers from 12 to 18 years old from two schools in San Lorenzo were included. The variables studied were: Age, Sex, vision less than 20/40, prior consultation history, family history of wearing glasses, electronic device usage hours, use of social networks, hours spent outdoors, time spent on leisure reading, presence of eye symptoms. Statistical analysis: SPSSv21 using descriptive and inferential statistics. Protocol approved by the institutional ethics committee with informed consent. Results: 300 adolescents were evaluated, the median age was 14 years, female sex was more prevalent $(58.7 \%) ; 7.3 \%$ presented with vision impairment, $64.3 \%$ had not consulted previously. A family history of wearing glasses was not significant. It was noted that the daily hours of use of electronic devices were greater in those with reduced vision with less time spent outdoors in those with impaired vision. The most frequent diagnosis was astigmatism. Conclusion: The frequency of vision impairment in adolescents was $7.3 \%$. Adolescents with impaired vision had more daily hours of using electronic devices, and less hours spent outdoors.

Keywords: Visión, electronic devices, refraction errors.

${ }^{1}$ Hospital General Pediátrico Niños de Acosta Ñu. San Lorenzo, Paraguay.

Correspondencia: Olivia Cardozo Correo: occs77@hotmail.com

Conflicto de Interés: Los autores declaran no poseer conflicto de interés

Recibido: 11/08/2019 Aceptado:25/10/2019

Doi: https://doi.org/10.31698/ped.46032019004

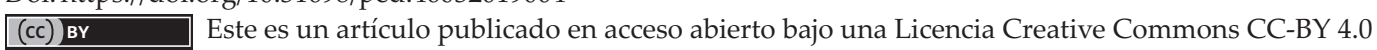




\section{INTRODUCCION}

La aparición de los dispositivos móviles digitales (teléfonos inteligentes $\mathrm{y}$ tablet) ha supuesto un cambio importantísimo en los hábitos de conducta y de comunicación de las personas y han convertido en el epicentro del interés de una gran mayoría de niños y jóvenes. En concreto, son las aplicaciones de mensajería instantánea y redes sociales las que polarizan su interés, hasta el punto que el VI Estudio anual de Redes Sociales, de enero de 2015, indica que el $97 \%$ de los jóvenes entre 14 y 17 años usa estas aplicaciones a diario ${ }^{(1)}$.

En Paraguay, el 77 \% de los niños y jóvenes que tienen entre 8 y 18 años cuentan con un aparato celular propio. El $14 \%$ usa el teléfono de sus padres, el $2 \%$ utiliza el de sus hermanos, el $2 \%$ usa el de algún amigo o compañero, y solo el $5 \%$ no utiliza aparatos electrónicos ${ }^{(2)}$. En el 2017 la Secretaría Nacional de Tecnologías de la Información y Comunicación (Senatics) realizó una encuesta sobre el uso de internet en Paraguay. Los datos fueron que el $43,2 \%$ de los encuestados declararon tener una conexión a internet en el hogar, mientras que el $94,2 \%$ comentó que tiene teléfonos celulares inteligentes (smartphones) ${ }^{(3)}$.

Los niños y adolescentes menores de 18 años representan aproximadamente uno de cada tres usuarios de internet en todo el mundo. Por otro lado los defectos de refracción no corregidos son la principal causa de discapacidad visual ${ }^{(4)}$ La OMS mediante la iniciativa del programa VISION 20/20, busca la disminución de la ceguera evitable a través de la detección precoz en la edad escolar. Teniendo en cuenta que para el desarrollo de los defectos refractivos influyen factores externos modificables, se debería tener como objetivo la prevención, atención y tratamiento oportuno ${ }^{(4)}$.

En la población entre 9 y 14 años con miopía, se ha reportado que el tiempo dedicado a la lectura y escritura se relaciona significativamente con la progresión de miopía ${ }^{(5)}$. En un estudio en adultos se encontró que la exposición al uso de computadora más de $30 \mathrm{~h} /$ semana incrementa el riesgo de desarrollo o progresión de la miopía ${ }^{(6)}$. También se corroboro una menor incidencia de desarrollo de miopía en jóvenes que realizaron actividades al aire $\operatorname{libre}^{(5)}$.

El objetivo del presente estudio fue determinar la frecuencia de alteraciones de la visión y su relación con la utilización de aparatos electrónicos en adolescentes de dos escuelas de San Lorenzo.

\section{MATERIALES Y MÉTODOS}

Estudio observacional, descriptivo, prospectivo, con componente analítico. Fueron incluidos Adolescentes de 12 a 18 años de edad de dos escuelas de San Lorenzo de octubre a diciembre del 2018. Se excluyeron aquellos que presentaban patologías oculares que podrían dificultar la realización del Test de Snellen. El muestreo fue no probabilístico de casos consecutivos. El Reclutamiento se realizó de la siguiente manera: se seleccionaron dos escuelas públicas de la Ciudad de San Lorenzo; la escuela Mcal. José Feliz Estigarribia Nro. 276 y la escuela San Antonio Fe y Alegría. Previa autorización de los directivos de dichos centros educativos, se solicitó el consentimiento informado por escrito a los padres o encargados y consentimiento verbal de los participantes, se recogieron los datos por medio de una encuesta cerrada que proporciono información sobre uso de aparatos electrónicos y síntomas oculares. Posteriormente una de las investigadoras midió la visión por medio de la Tabla de Snellen. La evaluación se realizó individualmente, ambos ojos por separado y con anteojos los que ya lo utilizaban. La aplicación de la Tabla de Snellen se realizó en una habitación con buena iluminación y ventilación y a 6 metros de distancia. Posteriormente los participantes con la prueba alterada, fueron citados al consultorio oftalmológico para su evaluación con el especialista. Las Variables estudiadas fueron: Edad, sexo, antecedentes de consulta previa, antecedente familiar, la visión, actividades al aire libre, tipo y tiempo de uso de aparato electrónico, horas de sueño, uso de redes sociales, síntomas oculares, dificultad para la visión lejana y cercana, tiempo dedicado a las tareas escolares y a la lectura. En relación al cálculo de tamaño de muestra, para un intervalo de confianza de $95 \%$ y una amplitud de 0.10 , se incluyeron a 300 adolescentes de 12 a 18 años 
de edad, que fueron seleccionados en forma aleatoria de las escuelas participantes. Los datos fueron analizados en el sistema SPSSV 21, las variables cualitativas, fueron expresadas en porcentajes y las cuantitativas en medianas con rangos intercuartilicos La relación entre variables cualitativas se realizó utilizando la prueba de Chi cuadrado y el Test de Fisher y entre las variables cuantitativas por la U de Mann Whitney. Se respetaron los principios éticos de Autonomía, ya que para el ingreso de los pacientes al estudio se solicitó el consentimiento informado por escrito, tanto de sus padres como de los adolescentes. Beneficencia, la inclusión de los pacientes en el estudio no constituyó ningún riesgo para los mismos. Justicia, todos los pacientes tuvieron la misma oportunidad de ingresar al estudio sin discriminación de ninguna clase. El protocolo fue aprobado por el Comité de Ética institucional.

\section{RESULTADOS}

Cumplieron los criterios de inclusión 300 adolescentes. Los datos demográficos, así como evaluación oftalmológica previa, antecedentes familiares y porcentaje de alteración del test de Snellen practicado en la escuela se encuentra en la Tabla 1.

Tabla 1. Características de la población de adolescentes participantes. N 300.

\begin{tabular}{lc}
\hline Edad (mediana-P25-P75) & $14(13-16)$ \\
\hline & $\mathbf{N}(\%)$ \\
\hline Sexo femenino & $176(58.7)$ \\
Sin evaluación oftalmológica previa & $194(64,6)$ \\
Test Snellen Alterado & $22(7,3)$ \\
Antecedentes familiares de utilización de anteojos & $104(34,6)$ \\
\hline
\end{tabular}

En el grupo de adolescentes que no habían tenido antes evaluación oftalmológica, tenían visión alterada en el 6,3\% (19/194), frente al 1\% (3/106) de aquellos que fueron evaluados alguna vez.

Los adolescentes con antecedentes familiares de uso de anteojos $(n=104)$, el 5,6\% (17/104) presentaron alteración de la visión frente al 1,6\% /5/114) en el grupo sin dichos antecedentes familiares $p=0,0001$ (Test de Fisher).
En cada grupo con y sin visión alterada detectada por la prueba de Snellen, se analizaron las horas de uso de diario de aparatos electrónicos, las dedicadas a tareas no académicas y horas al aire libre. Tabla 2.

Los 22 participantes con el test de Snellen alterado, 17 fueron evaluados por la oftalmopediatra. Las patologías encontradas están en la Tabla 3.

Tabla 2. Horas dedicadas al aire libre y a tareas fuera del horario académico. N=300.

\begin{tabular}{lccc}
\hline & Visión Normal $\mathbf{n}=\mathbf{2 7 8}$ & Visión alterada $\mathbf{n = 2 2}$ & $\mathbf{p}$ \\
\hline $\begin{array}{l}\text { Horas de uso aparatos electrónicos } \\
\text { Mediana (P25-P75) }\end{array}$ & $4(1-10)$ & $8(6-15)$ & $0,0001 \mathbf{1}$ \\
\hline $\begin{array}{l}\text { Tiempo de tareas no académicas } \\
\text { Mediana (P25- P75) }\end{array}$ & $2(1-5)$ & $3(1-5)^{1}$ & $0,0001 \mathbf{1}$ \\
\hline $\begin{array}{l}\text { Horas al aire libre } \\
\text { Mediana (P25- P75) }\end{array}$ & $4(1-10)$ & $2(1-4)^{1}$ & $0,0001 \mathbf{1}$ \\
\hline $1=$ U Mann Whitney & & &
\end{tabular}


Tabla 3. Diagnósticos en adolescentes evaluados en el servicio de oftalmología. $n=22$.

\begin{tabular}{lcc}
\hline & $\mathbf{n}$ & $\mathbf{\%}$ \\
\hline Astigmatismo & 6 & 27,2 \\
Miopía & 5 & 22,7 \\
Ambliopía & 2 & 9 \\
Toxoplasmosis activa & 1 & 4,5 \\
Sano & 3 & 13,6 \\
No Acude & 5 & 22,7 \\
\hline Total & $\mathbf{2 2}$ & $\mathbf{1 0 0}$ \\
\hline
\end{tabular}

Se indago sobre el uso de redes sociales. El 100\% de los adolescentes las utilizaba. El 68,3\% (190/278) tenían más de una, el 24\% (67/278) utilizaban solo WhatsApp, el 5,8\% (16/278) Facebook y el 1,7\% (5/278) Instagram.

\section{DISCUSIÓN}

Con este estudio la frecuencia de alteración de la visión diagnosticada mediante el Test de Snellen es ligeramente superior a lo reportado por Molina y colaboradores realizado en la escuela primaria Ignacio Agramonte en el 2015 donde informa un valor del 2 al $5 \%{ }^{(6)}$.

Se observó que aquellos adolescentes con alteración de la visión utilizaban más horas de aparatos electrónicos y más de una red social diariamente; este hallazgo es significativo comparando con aquellos que presentaban visión normal, esta cuestión se ajusta al VI estudio anual de redes sociales realizado en enero del 2015 donde se indica que el $97 \%$ de los jóvenes utilizan estas aplicaciones en forma diaria ${ }^{(1)}$

En un estudio realizado por la Senatic en donde se evaluó las costumbres de uso de TIC en adolescentes, se encontró que $77 \%$ de niños y jóvenes contaban con un celular propio ${ }^{(2)}$, en el presente estudio todos los pacientes que tenían la visión disminuida contaban también con un celular propio.

Aquellos adolescentes que tenían más actividades al aire libre presentaban menos síntomas oculares y visión normal comparando con aquellos que tenían menos actividades al aire libre, siendo este hallazgo estadísticamente significativo. Los síntomas oculares que se describen, se relacionan con el uso de la visión cercana. Este factor ha sido estudiado en diferentes investigaciones donde se demuestra una relación directa entre el tiempo dedicado a trabajos en visión próxima y la aparición de síntomas, especialmente en el inicio de miopía o de su progresión ${ }^{(6-8)}$. Se vio además que los adolescentes con visión disminuida dedicaban mayor cantidad de horas diarias a tareas fuera del horario académico,

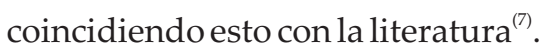

Se vio que la mediana de edad de los pacientes con alteración de la visión fue de 14 años, con un predominio del sexo femenino dato concordante con otros trabajos $^{(8)}$.

Un dato llamativo fue que 7 de cada 10 adolescentes no habían consultado con un oftalmólogo previamente. Ninguno de los adolescentes evaluados en el servicio de oftalmología tuvo anteriormente una evaluación oftalmológica, considerando que el hospital se encuentra en una zona cercana a la escuela y la consulta es gratuita. Esto evidencia la falta de control precoz, incluso existiendo antecedentes familiares de 1er grado con uso de anteojos hecho este que debería alertar a los padres para realizar una consulta antes de que existan síntomas. Los factores hereditarios influyen en el estado refractivo de los pacientes. En el presente estudio 1 de cada 3 pacientes tenia familiares con antecedente de uso de anteojos, cuyo dato es concordante con la literatura en cuanto a factores hereditarios ${ }^{(9)}$.

Aquellos detectados con visión disminuida presentaron en un alto porcentaje síntomas oculares, 
principalmente errores refractivos en el hallazgo oftalmológico que coincide con los trabajos reportados en donde los síntomas oculares se asocian con estos ${ }^{(8)}$. Se encontró que el defecto de refracción más frecuente fue el astigmatismo que coincide con Jiménez, Hernández, en un estudio sobre la ambliopía en escolares en el municipio de Caimito en Cuba del $2013^{(9)}$; también con Arellano y colaboradores en el artículo sobre problemas refractivos en estudiantes de medicina en Chimborazo- Ecuador del año $2013^{(7)}$.

Entre los diagnósticos encontrados, pudo detectarse como hallazgo casual un adolescente diagnosticado con toxoplasmosis activa en el ojo de menor visión. En un estudio previo realizado por una de las autoras, en el mismo hospital donde se realizó el presente trabajo, se encontró una prevalencia de toxoplasmosis ocular del $0,8 \%$ en un periodo de 3 años (2011 -2015), todos en etapa tardía y con graves lesiones de corioretinitis ${ }^{(10)}$.

Tras los diagnósticos establecidos se indicaron anteojos en los adolescentes que lo necesitaban. El paciente con toxoplasmosis activa se derivó al servicio de infectología para tratamiento y seguimiento conjunto con oftalmología.

\section{REFERENCIAS BIBLIOGRÁFICAS}

1. Pulido J, Blanco F. Estudio sobre el uso de dispositivos móviles en niños y adolescentes en Extremadura [Internet]. 2015 [citado ene 2013]. Disponible en: https://enmarchaconlastic.educarex.es/conectadoyseguro /pdf/encuestadispositivos.pdf

2. SENATIC's. Usos de costumbres de TIC en adolescentes [Internet]. SENATIC's; 2017. Disponible en: https://www.senatics.gov.py/observatorio/publicaciones

3. Capli, F, First Análisis y Estudios. Encuesta acceso y uso de internet Paraguay 2017 [Internet]. SENATIC's; 2017. Disponible en: https://www.senatics.gov.py/obser vatorio/publicaciones

4. UNICEF. Estado mundial de la infancia: niños en un
Este estudio tiene la limitación de incluir una muestra que no representa a la población de adolescentes del país. Es necesario realizar un estudio con mayor numero de pacientes, para una mejor evaluación del impacto de la utilización frecuente de los dispositivos electrónicos, sobre la salud visual Sin embargo, destaca la necesidad de detección de alteración de la visión por medio de medición de la agudeza visual a través del test de Snellen en las escuelas y colegios, que podría realizarse por los maestros, previa capacitación, con el fin de detección oportuna de los vicios de refracción en los niños y adolescentes, ya que se vio que la frecuencia de consulta para control oftalmológico es muy baja, a pesar de contar con un servicio de oftalmología en su localidad. La realización de tamizaje en las escuelas contribuirá a disminuir la incidencia de alteración en la visión.

\section{CONCLUSIÓN}

La frecuencia de alteración de la visión en los adolescentes es de 7,3\%. Los adolescentes con una visión alterada tuvieron más horas diarias de utilización de aparatos electrónicos, todos utilizaban más de una red social en forma diaria y se observó que la mediana de horas que pasaban al aire libre fue menor en los mismos.

mundo digital. New York: UNICEF; 2017.

5. Rey-Rodríguez DV, Álvarez-Peregrina C, MorenoMontoya J. Prevalencia y factores asociados a miopía en jóvenes. Revista Mexicana de Oftalmología. 2017; 91(5):223228. DOI: https://doi.org/10.1016/j.mexoft.2016.06.007

6. Molina D, Ruíz A, Valdés A, Rodríguez F. Comportamiento de los defectos refractivos en estudiantes de la escuela primaria Ignacio Agramonte y Loynaz. Cienfuegos. 2015; 15(2):202-209.

7. Arellano G, Chávez A, Arellano S, Chaves C. Estudio de la agudeza visual y problemas refractivos en estudiantes de medicina de la escuela superior politécnica de Chimborazo 2013. SCientifica 2014; 12 (1):25-32. 
8. Ruiz J, Madrid D, Barra B, Romero F. Prevalencia de errores refractivos en la población joven-urbana de Mozambique. Gaceta optometría. 2013;477:1-7.

9. Jiménez G, Hernández L, Soto M. Ambliopía en escolares del seminternado Juventud de Acero, de municipio de caimito. Revista Cubana de oftalmología.
2013;26(Suppl.1):593-604.

10. Cardozo O, Mesquita M, Godoy L. Toxoplasmosis ocular: Frecuencia y características clínicas en consultorio de oftalmología pediátrica. Pediatr. (Asunción). 2018; 45: 223-228. 